\title{
Immunohistochemical evidence of the co-localisation of cocaine and amphetamine regulatory peptide with neuronal isoform of nitric oxide synthase, vasoactive intestinal peptide and galanin within the circular muscle layer of the human caecum
}

\author{
M. Bulc ${ }^{1}$, S. Gonkowski ${ }^{1}$, P. Landowski², B. Kamińska², J. Całka ${ }^{1}$ \\ ${ }^{1}$ Department of Clinical Physiology, Faculty of Veterinary Medicine, University of Warmia and Mazury in Olsztyn, \\ Olsztyn-Kortowo, Poland \\ ${ }^{2}$ Department of Paediatrics, Gastroenterology, Hepatology and Nutrition, Medical University of Gdansk, Poland
}

[Received 30 September 2014; Accepted 10 November 2014]

The enteric nervous system consists of about one hundred million of neurons. In big mammals (including humans) intestinal enteric neuronal cells are grouped into three types of intramural ganglia located within myenteric, as well as outer and inner submucosal plexuses, which are connected by numerous nerve fibres. Both nerve fibres and cell bodies located in the gastrointestinal tract utilise a broad spectrum of active substances. One of them is cocaine- and amphetamine-regulated transcript peptide (CART). The goal of the current study was to determinate the distribution and degree of co-localisation of CART with substances taking part in intestinal motor activity by double labelling immunofluorescence technique. During the study CART-, neuronal isoform of nitric oxide synthase (nNOS)-, vasoactive intestinal peptide (VIP)- and/or galanin (GAL) — like immunoreactive (LI) nerve fibres in the circular muscle layer of the human caecum were observed in all patients studied. The degree of co-localisation of particular substances with CART depended on their type. The majority of CART-LI fibres contained simultaneously nNOS, slightly lower degree of co-localisation was observed in the case of the VIP, while simultaneously CART- and GAL-positive nerve fibres were observed less often. (Folia Morphol 2015; 74, 2: 176-182)

Key words: cocaine- and amphetamine-regulated peptide, neuronal isoform of nitric oxide synthase vasoactive intestinal peptide, galanin, caecum, human

\section{INTRODUCTION}

The gastrointestinal (GI) tract is involved in a lot of processes important for the living organism, such as transport of the chyme, secretion and absorption of ions, water and nutrients, as well as defence against pathogens. These functions are regulated both by extrinsic innervation [27] of intestines and the enteric nervous system (ENS), localised in the wall of GI tract from the oesophagus to the anus $[12,13]$. Three kinds of intramural ganglionated plexuses are distinguished in the construction of the ENS within small intestine of large mammals including human, 
i.e. myenteric plexus located between the longitudinal and circular muscle layers and submucosal plexus, outer submucosal plexus, situated close to the circular muscle layer and the inner submucosal plexus between the muscularis mucosa and lamina propria $[5,12]$. Furthermore, all three plexuses are connected through numerous nerve fibres, which are especially prolific in the circular muscle layer of intestinal wall [20]. Both cell bodies located in the enteric plexuses as well as nerve fibres connecting them use various active substances, which can play the role of neurotransmitters and/or neuromodulators [21].

One of them is cocaine- and amphetamine-regulated transcript peptide (CART). In spite of the fact that this substance was described for the first time over 30 years ago [28], its exact functions in the living organism are not yet entirely clear. CART was found in several systems and organs, among them in the central and peripheral nervous system, especially in the hypothalamus $[7,38]$. It must be emphasized that during the last years the number of structures, where the presence of CART has been confirmed has sharply increased and one of these systems, being rich in CART is the ENS [9].

Till now, several important functions of CART have been described, amongst which regulation of homeostasis including food intake, motor activity, endocrine regulation and stress response seem to be the most important [29]. However, the exact function of CART in the Gl tract remains unclear [8], because all known peripheral CART - induce effects, such as regulation of gastric acid secretion or contraction of intestinal muscles [38], may result from its central action. Previous studies on the digestive tract have described the numerous CART immunoreactive neuronal structures in the myenteric plexus and ileal circular muscle layer and may suggest that this peptide is involved in the regulation of intestinal motility [19]. Moreover, it is known that CART in enteric neurons often co-localises with other neuronal factors $[1,4]$, but many aspects of this co-localisation are unknown. So, the aim of the present study was to determine the degree of co-localisation of CART with vasoactive intestine polypeptide (VIP), neuronal isoform of nitric oxide synthase (nNOS - marker of nitrergic neurons) and galanin (GAL) in nerves within the circular muscle layer in the human caecum. VIP and nitric oxygen are known as relaxatory factors which inhibit the intestinal motility $[6,32,33]$, whereas the influence of GAL on intestinal muscles depends on the segment of $\mathrm{Gl}$ tract and animal species [23]. It should be pointed out that the knowledge about CART - immunoreactive enteric neuronal structures within human intestine is very scanty, especially in children $[16,22]$ and information about CART within the caecum in children is limited to 1 study [4]. On the other hand it is known that CART can be involved in the processes of the ontogenesis [26], and the caecum is the fragment of the digestive tract, where gut microbiota exists and various pathological processes can develop [11, 24]. So, the present investigation can be the introduction to further studies, which in the future will explain the functions of CART not only in physiological aging of human digestive tract, but also during pathological processes within the intestine.

\section{MATERIALS AND METHODS}

\section{Tissue preparation}

Fragments of the caecum were obtained from children ( 3 girls and 3 boys, at the age of 2-3 years) hospitalised in the Clinical Hospital of Medical University in Gdansk (Poland) just after the elimination of ileostomy, performed on the healthy segment of intestine during treatment of Hirschsprung's disease. The obtained samples have not shown any macroscopic and microscopic pathological changes. The size of collected tissue was dependent only on the surgery technique and performed immunohistochemical studies did not affect the decision of the surgeon concerning the size of resected caecum fragments. All medical procedures during the current study have been in accordance with ethical standards known as the Helsinki Declaration (revised 1983) and were conducted in compliance with the instruction of Bioethical Committee.

\section{Immunofluorescence technique}

Immediately after collection, fragments of caecum (about $1 \mathrm{~cm}$ in length) were fixed by immersion in a solution of $4 \%$ paraformaldehyde in $0.1 \mathrm{M}$ phosphate buffer ( $\mathrm{pH} \mathrm{7.4)}$ for $30 \mathrm{~min}$, rinsed several times with phosphate buffer, transferred into $18 \%$ sucrose solution and stored at $4^{\circ} \mathrm{C}$ until sectioning. Frozen tissue were cut in a cryostat Microm HM 560 cryostat (Carl Zeiss, Germany), into $10-\mu \mathrm{m}$-thick sections and mounted on chrome alun-coated slides. The sections were processed for double-immunofluorescence stainings. Briefly, after air-drying at room temperature for $45 \mathrm{~min}$ and rinsing in $0.1 \mathrm{M}$ phosphate-buffered saline (PBS; pH 7.4; $3 \times 10 \mathrm{~min}$ ), the sections were 
incubated in a blocking buffer containing: $10 \%$ of normal goat serum (MP Biomedicals, USA), in $0.1 \mathrm{M}$ phosphate buffer saline (PBS), $0.1 \%$ donkey serum (Abcam, UK), 1\% Triton X-100 (Sigma-Aldrich, USA), $0.05 \%$ Thimerosal (Sigma-Aldrich, USA) and $0.01 \% \mathrm{NaN} 3$ for $1 \mathrm{~h}$ at room temperature to reduce non-specific background staining. Subsequently, after another wash in PBS ( $3 \times 10 \mathrm{~min})$, the sections were incubated overnight at room temperature with primary antibodies directed towards CART (mouse monoclonal; R\&D System, Mineapolis, MN, USA, cat. No. MAB 163; working dilution 1: 1000), VIP (rabbit polyclonal; Biomol, Hamburg, Germany, cat. No. VA1285; working dilution 1: 6000), GAL (rabbit polyclonal: Millipore, Billerica, MA, USA, cat. No. $A B$ 2233; working dilution 1:1000) and nNOS (rabbit polyclonal: Chemicon, Billerica, MA, USA, cat. No. AB 5380; working dilution 1:4000). On the following day, the sections were rinsed and incubated with secondary antibodies conjugated with Alexa fluor 488 (donkey anti mouse: Molecular probes, cat. No. A21202, working dilution 1:1000) or Alexa fluor 546 (donkey anti rabbit: Molecular probes, cat. No. A10040, working dilution 1:1000). Then, the sections were rinsed 3 times (PBS, $3 \times 5 \mathrm{~min}$ ) and covered with polyethylene glycol/glycerine solution. The prepared specimens were viewed and photographed using an Olympus BX51 microscope, equipped for appropriate epi-illumination fluorescence microscopy filter sets. Pictures were captured with monochrome camera (Olympus XM 10) and analysed with Cell F software (Olympus, Tokyo, Japan).

Negative controls employed in the immunofluorescence procedure included pre-absorption test. The pre-absorption test was performed as follow: sections of the caecum were incubated with "working" dilutions of primary antibodies directed toward CART, nNOS, VIP, GAL, which had been preabsorbed for $18 \mathrm{~h}$ at $37^{\circ} \mathrm{C}$ with $20 \mu \mathrm{g}$ of appropriate purified protein CART (003-63, Phoenix Pharmaceuticals), VIP (064-24, Phoenix Pharmaceutical), GAL (026-06, Phoenix Pharmaceutical) and nNOS (N3033, Sigma, St Louis, MO, USA). Additional negative controls such as omission and replacement of all primary antisera with nonimmune sera were also performed.

Nerve profiles were estimated in 4 section per patient (in 5 fields per section). For semi quantitative evaluation of the density of nerves immunoreactive to each substance studied, an arbitrary scale was used, where $(-)$ means the absence of fibres $(+)-$ single fibres, $(++)-$ rare nervous fibres, $(+++)-$ numerous fibres and $(++++)-$ very dense nervous processes. The same method of analysis was used for measuring the degree of co-localisation of CART with each of other neuronal substances studied.

\section{RESULTS}

Immunofluorescence staining showed the presence of numerous CART-like immunoreactive (LI) nerve fibres, which were evenly distributed throughout the all circular muscle layer of the human caecum. Structurally CART-positive nerve fibres comprise diverse population with several kinds of nerves processes. The majority of them are long and thick, with strong level of immunoreactivity (Fig. 1A-C). These fibres were characterised by straight or curly course (Fig. 1A) as well as the presence or absence of varicosities (Fig. 1B, C). Moreover, short type of CART-LI nerve fibres with weaker level of immunoreactivity were noted during the present study and in this kind of processes varicosities were no observed (Fig. 1B).

Other neuronal factors studied were also visible in the human caecum, and the density of nerve fibres immunoreactive to each of them was different (Table 1). Namely, nerve fibres immunoreactive to nNOS were numerous and the majority of them were relatively long, thick (Fig. 1E, H), and often formed fibre bundles with sinuous course (Fig. 1H), although some nNOS-LI processes were short and thin (Fig. 1E). Nerve processes immunoreactive to VIP were also numerous, but in contrast to nNOS-LI fibres, in the vast majority they were short and thick (Fig. 2B, H). Only occasionally very long but delicate VIP-positive nerve fibres were noted (Fig. 2E). GAL-positive fibres were fewer then nerve processes immunoreactive to nNOS and VIP (Fig. 3B, E, H). They created long and delicate structures (Fig. 3B), which often formed fibre bundles (Fig. 3E). During the present study the co-localisation of CART with all substances studied was observed although the degree of coexistence of particular factors in the same fibres showed significant differences (Table 2) The vast majority of nNOS-positive fibres were simultaneously immunoreactive to CART (Fig. 1F, I), and nNOS+/ /CART- nerve processes were observed sporadically. The degree of co-localisation of CART with VIP and CART with GAL was slightly lower than concomitance of CART and NOS and similar to each other. The only difference was that the presence of GAL+/CART- nerve fibres (Fig. 3G-I), whereas VIP+/CART- processes were not studied (Fig. 2A-I). 

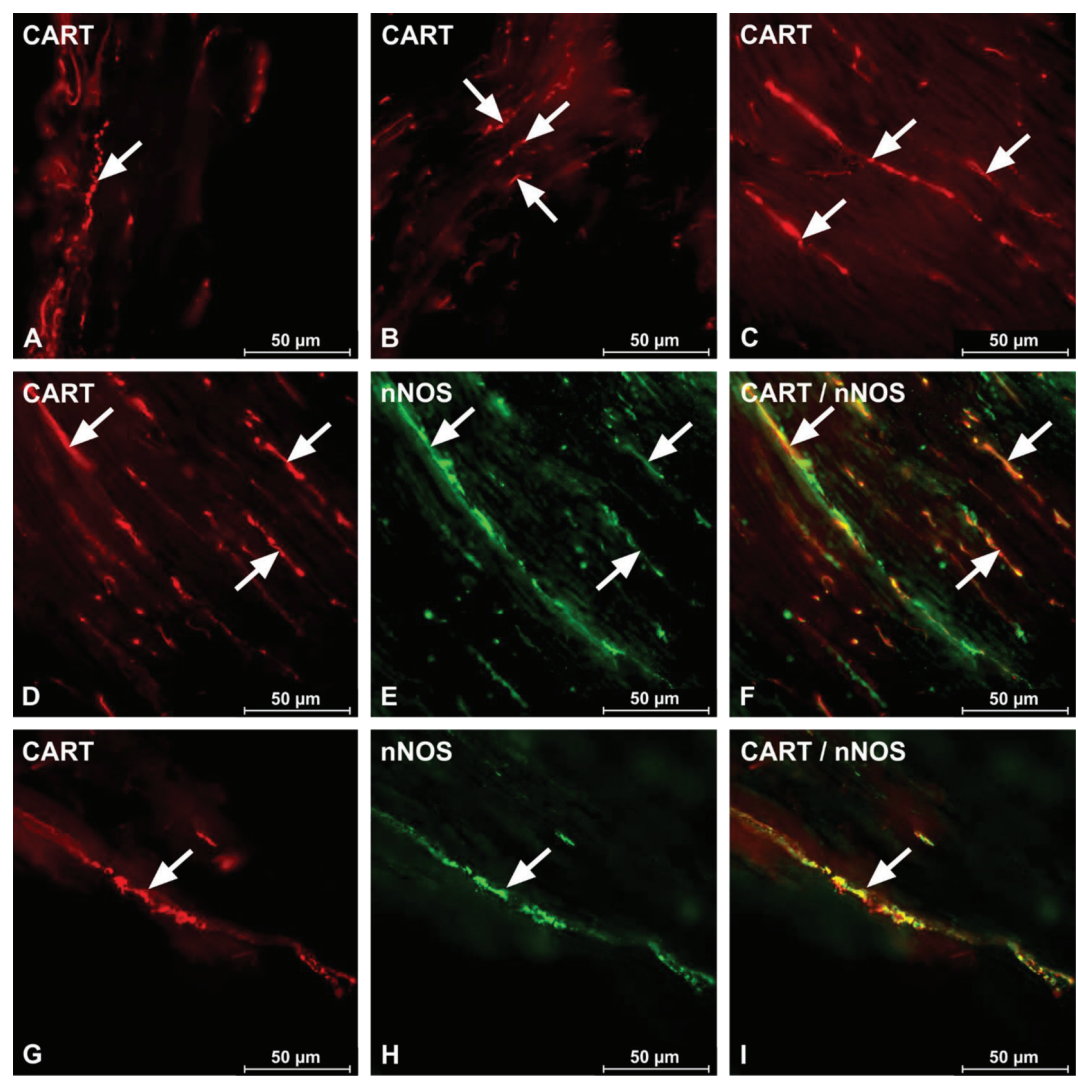

Figure 1. Fluorescence photograph showing nerve fibres immunoreactive to cocaine and amphetamine regulatory peptide transcript (CART) $(\mathbf{A}-\mathbf{C})$ and nitric oxide synthase (nNOS) (E, H) within the circular muscle layer of the human caecum. Panels D-I show co-localisation CART and nNOS. Arrows show nerve processes (left and medium column) immunopositive for a single antigens and co-localisation of both antigens (right column).

Table 1. Density of nerve processes in the circular muscle layer of human caecum immunoreactive for CART, nNOS, VIP, and GAL

\begin{tabular}{lc}
\hline Substance & Density of nerve fibres \\
\hline CART & ++++ \\
nNOS & ++++ \\
VIP & +++ \\
GAL & +++ \\
\hline
\end{tabular}

CART — cocaine and amphetamine regulatory transcript peptide; GAL — galanin; nNOS — nitric oxide synthase; VIP — vasoactive intestinal peptide

In the present study, visible differences between particular patients both in the density of fibres immunoreactive to neuronal factors studied and the degree of co-localisation of CART with other substances were not observed.

Moreover, all standard controls of antibodies i.e. pre-absorption, omission and replacement completely eliminated specific staining.

\section{DISCUSSION}

The obtained results show that CART is expressed in a large number of nerve fibres in the circular muscle layer of human caecum. It is in accordance with previous studies, where the presence of CART-positive neuronal structures was described within the gastrointestinal tract of many mammalian species including human [16, 19, 22], rat [9], pig [17, 34, 36, 37], sheep [1] and guinea pig [10], not only within nerve fibres, but also in neuronal cell bodies of enteric plexuses $[1,34,37]$. It should be pointed out that the knowledge about distribution and exact functions of CART in human intestine, especially in physiological states is very scanty, but results obtained during the present study are in accordance with previous sparse studies [4, 22].

Till now CART-positive nervous structures are observed primarily within the smooth muscle layer in all segments of the GI tract. CART-LI muscular nerve fibres and neuronal cells located in the myenteric plexus were noted for example in human descending colon [19], as well as all fragments of rat's and porci- 

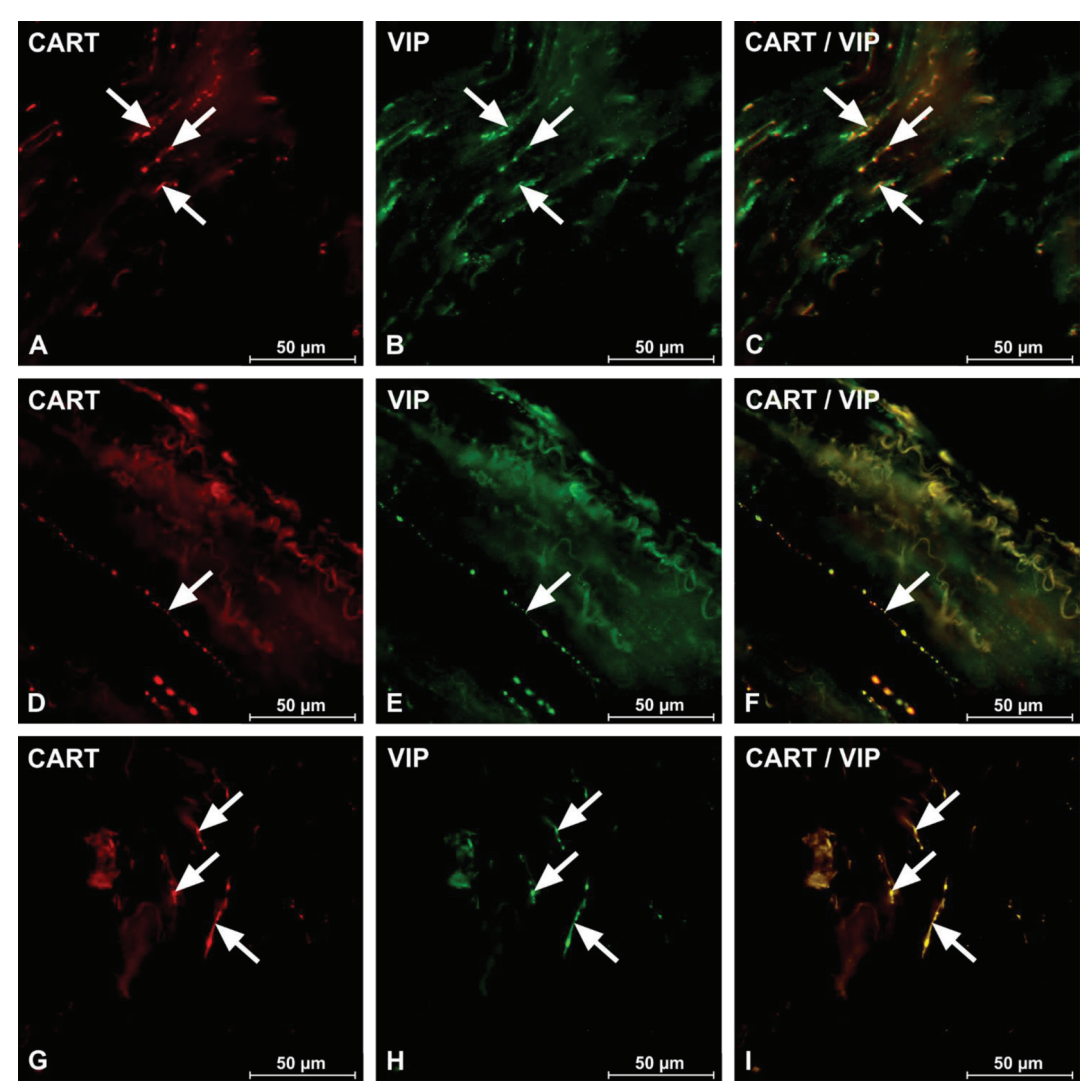

Figure 2. Co-localisation cocaine and amphetamine regulatory transcript peptide (CART) and vasoactive intestinal peptide (VIP) within the circular muscle layer of the human caecum (A-I). Arrows show nerve processes (left and medium column) immunopositive for a single antigens and co-localisation of both antigens (right column).

ne GI tract $[9,17,34,37]$. Unlike the circular muscle layer the number of CART-positive nervous structures within submucosal enteric ganglia and lamina were scanty [17, 34, 37], except large intestine of guinea pig, where CART-LI neurons and nerve fibres were abundant both in the submucosal and myenteric plexuses [10]. Nevertheless, previous investigations, as well as the results of this study confirmed that CART is located primarily within the muscle layer of the Gl tract in most of the mammals.

Despite numerous studies involving morphological, immunohistochemical or electrophysiological methods, exact function of the CART in the Gl tract is not fully understood [8, 23, 34]. CART is probably involved in the motility processes, but previous studies in vitro did not fully confirm this hypothesis. Namely, in a study conducted by Ekblad [8] author demonstrated that CART reduces nitric oxide donor-induced relaxations of ileal muscle strips, but the presence of CART did not induce any contractile or relaxatory responses of intestinal muscles. Moreover, relatively well-known effect of CART on delaying of gastric empting and reduction of food intake is probably the result of its central action [31]. It seems to be likely that CART may also play a modulatory functions in intestinal nervous transmission by the influence of other neuronal factors. The present results, which showed a considerable degree of co-localisation of CART with nNOS and VIP seem to confirm this thesis. Nitric oxide and VIP, previously described in $\mathrm{Gl}$ tract in various species $[6,14,25]$, are a pivotal non-adrenergic, non-cholinergic inhibitory neurotransmitters, which are involved in relaxation of intestinal muscles and play important roles in the regulation gut motility [3].

It is generally accepted that one neuron can contain some active substances and such substances, which co-localise in one neuronal cells often play similar roles within the digestive tract. So, one important method of getting to know CART function in the ENS are studies on the co-localisation of this peptide with other, better known active substances in the same 

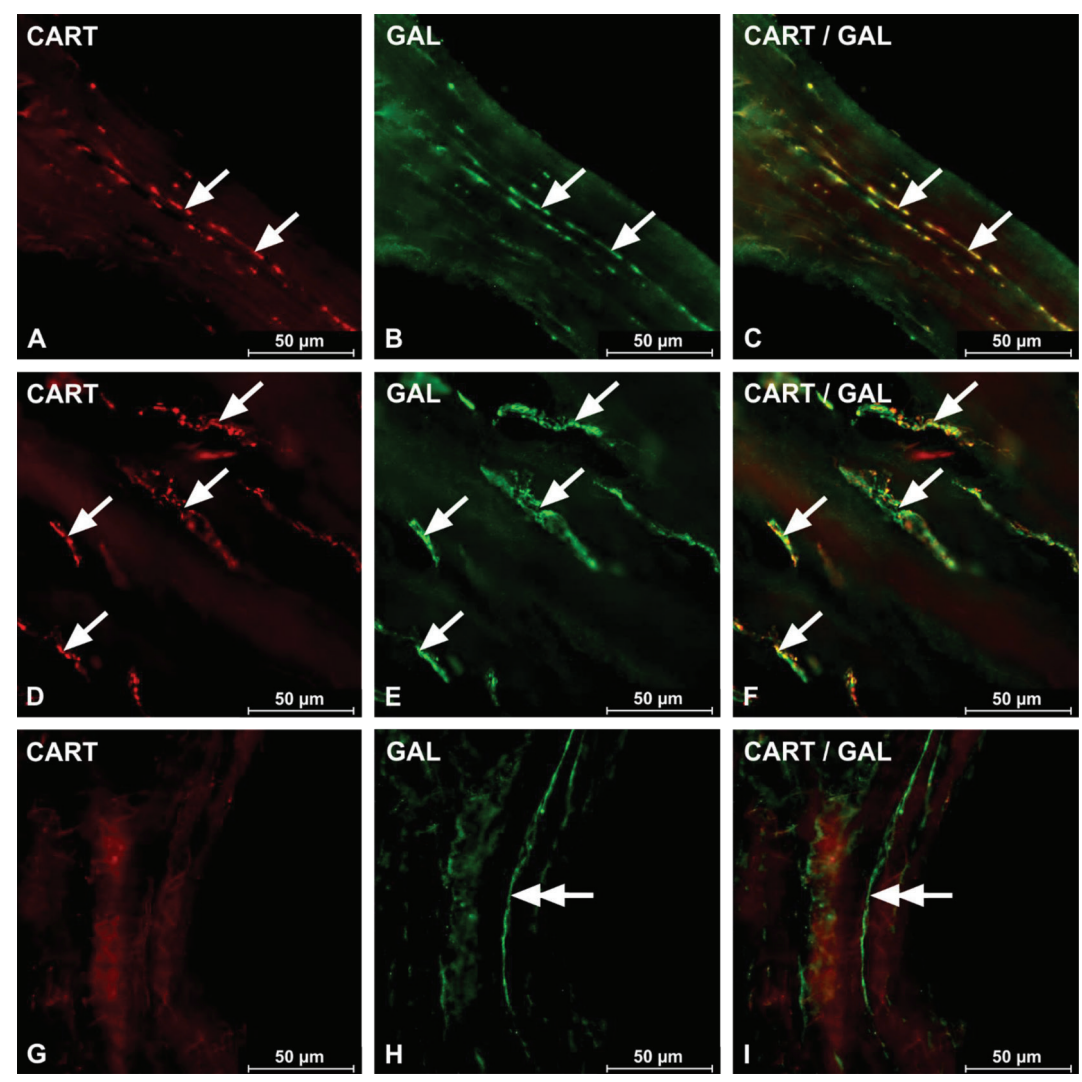

Figure 3. Co-localisation cocaine and amphetamine regulatory transcript peptide (CART) and galanin (GAL) within the circular muscle layer of the human caecum (A-F). Arrows show nerve processes (left and medium column) immunopositive for a single antigens and co-localisation of both antigens (right column). Double arrows show nerve fibres immunoreactive to $G A L(\mathbf{H}, \mathbf{I})$ and simultaneously CART negative (G, I).

Table 2. Degree of co-localisation of CART with other active substances within nerve fibres in the circular muscle layer of human caecum

\begin{tabular}{lc}
\hline Substances & Degree of co-localisation \\
\hline CART/nNOS & +++ \\
CART/VIP & ++ \\
CART/GAL & + \\
\hline
\end{tabular}

Abbreviations as in Table 1

neuronal structures. Results of the present study concerning the co-localisation of CART with VIP and nNOS, are in agreement with previous studies, where such co-localisation has been described in human descending colon [19], as well as in the GI tract of pig [17, 34, 36] rat [8] and sheep [1]. They also suggest that CART may play the role of co-transmitter or neuromodulator and can induce VIP and/or NO release at the presynaptic level [35]. Similar situation may affect the correlation between CART and GAL. Till now, galanin was described within the Gl tract of numerous mammals, such as human [19], rat [2], guinea-pig [10], pig [18, 36] dog $[15,33]$, and mouse [23]. Nonetheless, the influence of GAL on intestinal motility is not explicit and clearly depends on the segment of $\mathrm{Gl}$ tract and species studied $[19,30,33]$. Till now, the co-localisation of GAL with CART was described only in human descending colon, where it was slightly lower [18] in comparison with results in caecum, obtained during the present study. This fact confirms that GAL can play different roles in various parts of the $\mathrm{Gl}$ tract.

\section{CONCLUSIONS}

To sum up, results of this study have shown that CART is a relatively abundant neuronal factor in nerve fibres within the circular muscle layer of human caecum and it can co-localise with nNOS, VIP and GAL, what suggests similar roles all mentioned above substances. However, the exact function of this peptide within intestinal muscles remains unclear. Most likely, CART by interaction with another substances may act as a co-transmitter and/or neuromodulator, but accurate confirmation of this hypothesis needs further studies. 


\section{REFERENCES}

1. Arciszewski MB, Barabasz S, Skobowiat C, Maksymowicz W, Majewski M (2009) Immunodetection of cocaine- and amphetamine-regulated transcript in the rumen, reticulum, omasum and abomasum of the sheep. Anat Histol Embryol, 38: 62-67.

2. Bishop AE, Polak JM, Bauer FE, Christofides ND, Carlei F, Bloom SR (1986) Occurrence and distribution of a newly discovered peptide, galanin, in the mammalian enteric nervous system. Gut, 27: 849-857.

3. Brookes SJ (1993) Neuronal nitric oxide in the gut. J Gastroenterol Hepatol, 8(6):590-603.

4. Bulc M, Gonkowski S, Landowski P, Kamińska B, Całka J (2014) Immunohistochemical distribution of cocaine and amphetamine regulatory peptide-like immunoreactive (CART-LI) nerve fibers in the circular muscle layer and their relationship to other peptides in the human caecum. Acta Histochem, 116: 1029-1036.

5. Costa M, Glise H, Sjodahl R (2000) The enteric nervous system in health and disease. Gut, 47: Suppl. 4.

6. Chino Y, Fujimura M, Kitahama K, Fujimiya M (2002) Colocalization of NO and VIP in neurons of the submucous plexus in the rat intestine. Peptides, 23: 2245-2250.

7. Dylag T, Kotlinska J, Rafalski P, Pachuta A, Silberring J (2006) The activity of CART peptide fragments. Peptides, 27: 1926-1933.

8. Ekblad E (2006) CART in the enteric nervous system. Peptides, 27: 2024-2030.

9. Ekblad E, Kuhar M, Wierup N, Sundler F (2003) Cocaine- and amphetamine-regulated transcript: distribution and function in rat gastrointestinal tract. Neurogastroenterol Motil, 15: 545-557.

10. Ellis LM, Mawe GM (2003) Distribution and chemical coding of cocaine- and amphetamine-regulated transcript peptide (CART)-immunoreactive neurons in the guinea pig bowel. Cell Tissue Res, 312: 265-274.

11. Esaki M, Matsumoto T, Fuyuno Y, Maehata Y, Kochi S, Hirahashi M, lida M (2009) Giant inflammatory polyposis of the cecum with repeated intussusception in ulcerative colitis: report of a case. Am J Gastroenterol, 104: 2873-2874.

12. Furness JB (2012) The enteric nervous system and neurogastroenterology. Nat Rev Gastroenterol Hepatol, 69: 286-294.

13. Furness JB, Callaghan BP, Rivera LR, Cho HJ (2014) The enteric nervous system and gastrointestinal innervation: integrated local and central control. Adv Exp Med Biol, 817: 39-71.

14. Gaumnitz E, Sweet MA, Sengupta A, Singaram C (1995) Nitrinergic and peptidergic innervations and their inter-relationships in human colon. Neuropeptides, 29: 1-9.

15. Gonda T, Daniel EE, McDonald TJ, Fox JE, Brooks BD, Oki M (1989) Distribution and function of enteric GAL-IR nerves in dogs: comparison with VIP. Am J Physiol, 256 (5 Part 1): G884-G896.

16. Gonkowski S, Kamińska B, Burliński P, Kroll A, Całka J (2009) The influence of drug resistant ulcerative colitis on the numer of cocaine- and amphetamine-pegulated transcirpt peptide like immunoreactive (CART-LI) mucosal nerve fibers of the descending colon in children. Przegl Gastroenterol, 4: 147-151.

17. Gonkowski S, Burliński P, Skobowiat C, Majewski M, Arciszewski MB, Radziszewski P, Całka J (2009) Distribution of cocaine- and amphetamine-regulated transcript-like immunoreactive (CART-LI) nerve structures in the porcine large intestine. Acta Vet Hung, 57: 509-520.

18. Gonkowski S1, Burliński P, Skobowiat C, Majewski M, Całka J (2010) Inflammation- and axotomy-induced changes in galanin-like immunoreactive (GAL-LI) nerve structures in the porcine descending colon. Acta Vet Hung, 58: 91-103.

19. Gonkowski S, Kamińska B, Landowski P, Całka J (2013) Immunohistochemical distribution of cocaine- and amphetamine-regulated transcript peptide-like immunoreactive (CART-LI) nerve fibers and various degree of co-localization with other neuronal factors in the circular muscle layer of human descending colon. Histol Histopathol, 28: 851-858.

20. Hansen MB (2003) The enteric nervous system I: organisation and classification. Pharmacol Toxicol, 92: 105-113.

21. Hansen MB (2003) The enteric nervous system II: gastrointestinal functions. Pharmacol Toxicol, 92: 249-257.

22. Kasacka I, Piotrowska Z, Car H, Janiuk I, Lebkowski W (2012) Cocaine- and amphetamine-regulated transcript: identification and distribution in human gastrointestinal tract. J Biol Regul Homeost Agents, 26: 419-428.

23. Melander T, Hökfelt T, Rökaeus A, Fahrenkrug J, Tatemoto K, Mutt V (1985) Distribution of galanin-like immunoreactivity in the gastro-intestinal tract of several mammalian species. Cell Tissue Res, 239: 253-270.

24. Mondal SK (2009) Primary squamous cell carcinoma of the cecum. J Cancer Res Ther, 5: 328-330.

25. Palus K, Rytel L (2013) Co-localisation of cocaine- and amphetamine-regulated transcript peptide and vasoactive intestinal polypeptide in the myenteric plexus of the porcine transverse colon. Folia Morphol, 72: 328-332.

26. Risold PY, Bernard-Franchi G, Collard C, Jacquemard C, La Roche A, Griffond B (2006) Ontogenic expression of CART-peptides in the central nervous system and the periphery: a possible neurotrophic role? Peptides, 27: 1938-1941.

27. Skobowiat C, Gonkowski S, Calka J (2010) Phenotyping of sympathetic chain ganglia (SChG) neurons in porcine colitis. J Vet Med Sci, 72: 1269-1274.

28. Spiess J, Villarreal J, Wale W (1981) Isolation and sequence analysis of a somatostatin like peptide from ovine hypothalamus. Biochemistry, 20: 1982-1988.

29. Stanley SA, Small CJ, Murphy KG, Rayes E, Abbott CR, Seal U, Morgan DG, Sunter D, Dakin CL, Kim MS, Hunter R, Kuhar M, Ghatei MA, Bloom SR (2001) Actions of cocaine- and amphetamine-regulated transcript (CART) peptide on regulation of appetite and hypothalamo-pituitary axes in vitro and in vivo in male rats. Brain Res, 893: 186-194.

30. Tamura K, Palmer JM, Wood JD (1987) Galanin suppresses nicotinic synaptic transmission in the myenteric plexus of guinea-pig small intestine. Eur J Pharmacol, 136: 445-446.

31. Thim L, Kristensen P, Larsen PJ, Wulff BS (1998) CART, a new anorectic peptide. Int J Biochem Cell Biol, 30: 1281-1284.

32. Timmermans JP, Barbiers $M$, Scheuermann DW, Bogers JJ, Adriaensen D, Fekete E, Mayer B, Van Marck EA, De Groodt-Lasseel MH (1994) Nitric oxide synthase immunoreactivity in the enteric nervous system of the developing human digestive tract. Cell Tissue Res, 275: 235-245.

33. Wang YF, Mao YK, Fox-Threlkeld JE, McDonald TJ, Daniel EE (1998) Colocalization of inhibitory mediators, NO, VIP and galanin, in canine enteric nerves. Peptides, 19: 99-112.

34. Wojtkiewicz J, Gonkowski S, Bladowski M, Majewski M (2012) Characterisation of cocaine- and amphetamine- regulated transcript-like immunoreactive (CART-LI) enteric neurons in the porcine small intestine. Acta Vet Hung, 60: 371-381.

35. Van Geldre LA, Lefebvre RA (2004) Interaction of NO and VIP in gastrointestinal smooth muscle relaxation. Curr Pharm Des, 10: 2483-2497.

36. Zacharko-Siembida A, Valverde Piedra JL, Szymańczyk S, Arciszewski MB (2013) Immunolocalization of NOS, VIP, galanin and $\mathrm{SP}$ in the small intestine of suckling pigs treated with red kidney bean (Phaseolus vulgaris) lectin. Acta Histochem, 115: 219-225.

37. Zacharko-Siembida A, Arciszewski MB (2014) Immunoreactivity to cocaine- and amphetamine-regulated transcript in the enteric nervous system of the pig and wild boar stomach. Anat Histol Embryol, 43: 48-55.

38. Zhang M, Han L, Xu Y (2012) Roles of cocaine- and amphetamine-regulated transcript in the central nervous system. Clin Exp Pharmacol Physiol, 39: 586-592. 